'Grande Ospedale Metropolitano Niguarda, Milán, Italia.

${ }^{2}$ Cardiologia Hospital Sótero del Río/Pontificia Universidad Católica de Chile, Santiago, Chile. ${ }^{3}$ Unidad de Cardiología Intervencional y Hemodinamia, Hospital Sótero del Río, Santiago, Chile.

${ }^{4}$ Cardiología Clínica Universidad de los Andes, Santiago Chile. aResidente Cardiología Intervencional.

Trabajo no recibió financiamiento Los autores declaran no tener conflictos de interés.

Recibido el 7 de mayo de 2019, aceptado el 17 de octubre de 2019.

Correspondencia a: Dr. Nicolás Veas P. Unidad de Cardiología Intervencional y Hemodinamia Hospital Sótero del Río Avenida Concha y Toro № 3459 Puente Alto, Santiago, Chile. nicoveas@gmail.com

\section{Implante concomitante de MitraClip y cierre de orejuela izquierda: importancia de la punción transeptal y sus implicancias técnicas. Caso clínico}

\author{
NICOLÁS VEAS ${ }^{3,4}$, RENÉ HAMEAU ${ }^{2}$, STEFANO NAVA ${ }^{1}$, \\ JOSÉ WINTER $^{3}$, FRANCESCO SORIANO ${ }^{1}$, JACOPO OREGLIA ${ }^{1}$ \\ Concomitant deployment of MitraClip \\ devices and left atrial appendage closure. \\ Report of one case
}

We report a 65-years old woman with a history of permanent atrial fibrillation with high risk for ischemic and bleeding events. She developed a heart failure with severely impaired left ventricular ejection fraction and severe secondary mitral regurgitation. Given her high surgical risk, using transesophageal echocardiography guidance, a concomitant deployment of two MitraClip devices using a high-posterior septal puncture and a left atrial appendage closure with an Amplatzer Amulet occluder were performed through the same access.

(Rev Med Chile 2019; 147: 1350-1354)

Key words: Atrial Appendage; Atrial Fibrillation; Heart Valve Prosthesis Implantation.
E xiste una asociación fisiopatológica entre insuficiencia mitral (IM) y fibrilación. auricular (FA) en estados avanzados de la enfermedad. Así, no es infrecuente que la resolución quirúrgica de esta valvulopatía se suela asociar al cierre quirúrgico de la orejuela izquierda ${ }^{1}$. Por otro lado, recientemente hemos sido testigos del desarrollo de numerosas terapias de abordaje percutáneo. El dispositivo para reparación mitral percutánea MitraClip (Abbott Vascular, Santa Clara, CA) ha demostrado su eficacia y seguridad para el manejo de pacientes de muy alto riesgo con insuficiencia mitral degenerativa o funcional en pacientes seleccionados ${ }^{2,3}$.

A su vez, el cierre percutáneo de la orejuela izquierda (OI) es una terapia con una recomendación IIb por las guías AHA/ACC/HRS 2019 para pacientes con FA no valvular con riesgo aumentado de eventos isquémicos y que tienen contraindicación para anticoagulación a largo plazo $^{4}$.
Dado que ambas técnicas requieren abordajes similares mediante una punción transeptal, es posible plantear la posibilidad de realizarlas combinadamente en una misma intervención y teóricamente disminuir sus riesgos asociados.

A continuación, presentamos el caso clínico de una paciente con alto riesgo quirúrgico que requiriró el implante percutáneo concomitante del dispositivo MC sumado al cierre percutáneo de la orejuela izquierda (LAAC) con el sistema Amplatzer Amulet (Abbott Vascular, Santa Clara, CA, USA).

\section{Caso clínico}

Mujer de 65 años con antecedentes mórbidos de hipertensión arterial, enfermedad renal crónica etapa IV, cardiopatía coronaria revascularizada con angioplastía a la arteria descendente anterior asociada a enfermedad difusa en los otros vasos, FA permanente en terapia anticoagulante oral con 
INR de difícil control y posteriormente suspendida por hemorragia digestiva alta (úlcera duodenal) recurrentes. Su score $\mathrm{CHA}_{2} \mathrm{DS}_{2}$ VASCr era de 4 puntos y un HAS-BLED de 5 puntos.

Refiere historia de aproximadamente 12 meses de deterioro progresivo de su capacidad funcional con disnea de esfuerzos (NYHA 3). Ecocardiograma de superficie evidencia un ventrículo izquierdo dilatado (volumen de fin de diástole: $123 \mathrm{ml}$ $\left(79 \mathrm{ml} / \mathrm{m}^{2}\right)$ ) con disfunción sistólica severa (FEVI $30 \%$ ), válvula mitral con velos engrosados e insuficiencia severa funcional (vena contracta $9 \mathrm{~mm}$ ), diámetro del anillo en el límite superior, insuficiencia tricuspídea leve a moderada con PSAP de $31 \mathrm{mmHg}$ y ventrículo derecho levemente dilatado con función normal. Se optimiza terapia médica con mala respuesta por lo que se re-hospitaliza por insuficiencia cardiaca con NT-pro-BNP de 3.000 $\mathrm{pg} / \mathrm{ml}$, asociada a falla renal.

La paciente presenta un alto riesgo quirúrgico (score STS 13,8\% y Euroscore II 10,18\%). Por lo anterior, se plantea una resolución percutánea con implante de $\mathrm{MC}$, debido al alto riesgo de eventos isquémicos y hemorrágicos por la $\mathrm{FA}$, se plantea un LAAC con el dispositivo Amplatzer Amulet.

El ecocardiograma transesofágico (ETE) evidencia calcificación del anillo mitral con movimiento restrictivo principalmente del velo posterior y alteraciones degenerativas de ambos velos con regurgitación severa principalmente central y de menor cuantía medial (EROA $0.4 \mathrm{~cm}^{2}$, VR $36 \mathrm{ml}$ ), dilatación del anillo (antero posterior $36 \mathrm{~mm}$ ) con criterios de factibilidad técnica para un MC (velo posterior $13 \mathrm{~mm}$, largo de coaptación

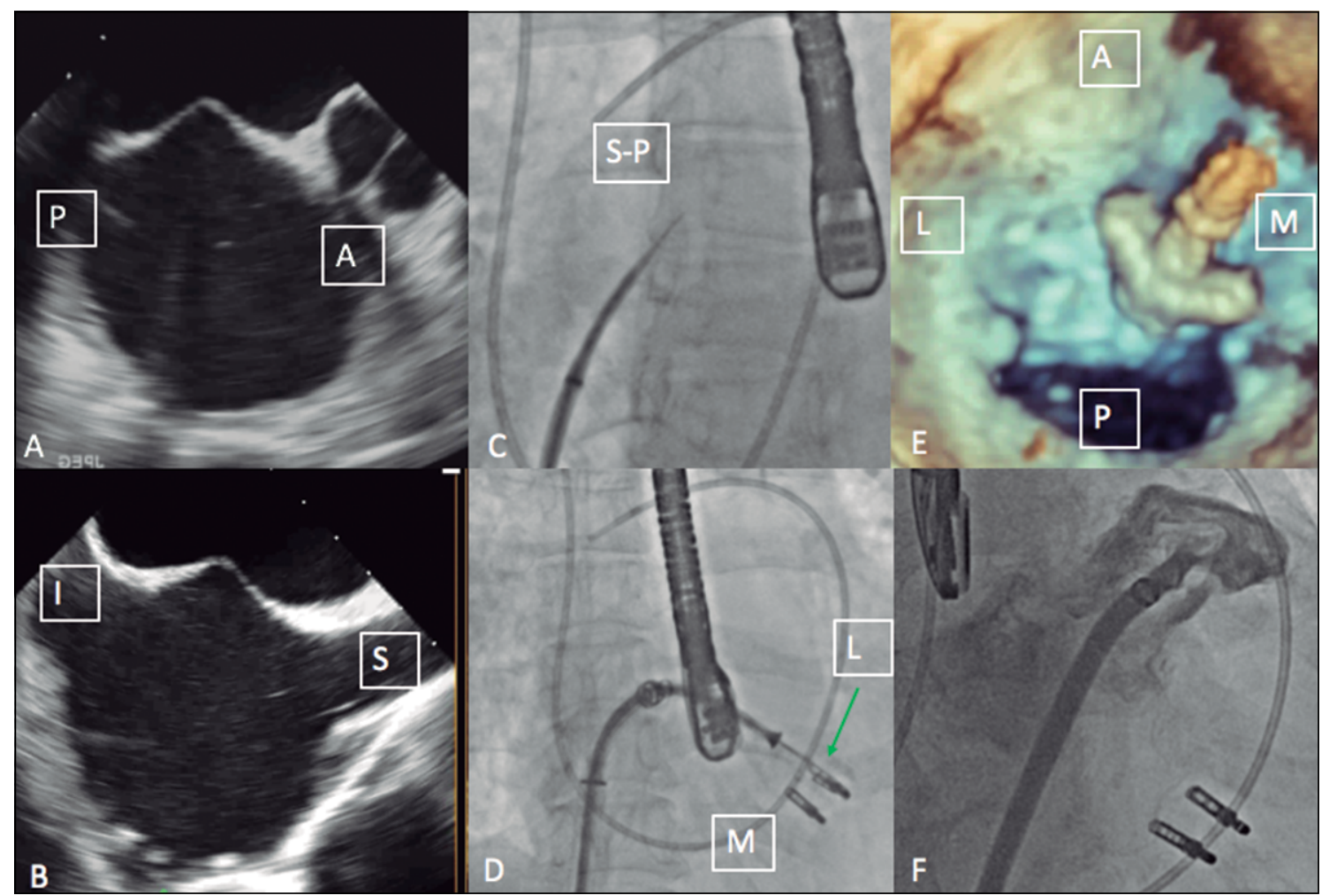

Figura 1. Punción transeptal e implante de MC. A: Imagen ETE eje corto: Nos muestra el septum interatrial, destaca la posición anterior (A) en relación a la aorta, tenting con la aguja de punción transeptal y la posición posrterior del septum (P). B: Imagen ETE transcaval: donde se visualiza el parte superior (S) e inferior (I) del septum interatrial con el respectivo tenting con la aguja de punción transeptal. C: Visión angiográfica que muestra el momento de la punción transeptal en localización superior posterior (S-P) recomendado para este tipo de procedimientos. D: Imagen angiográfica en el momento de la liberación del segundo Mitraclip en porción lateral. E: Imagen ETE 3D del plano valvular mitral. Destaca la relación anatómica al momento del implante del primer Mitraclip. "A" para anterior, "P" posterior, "L" lateral y "M" medial. F: Angiografía de la Ol e evaluación de la coaxiliadad para el implante de dispositivo Amplatzer. 
de $3,5 \mathrm{~mm}$, área valvular de $5 \mathrm{~cm}^{2}$, altura máxima de fosa oval con respecto al punto de coaptación del velo mitrálico de 4 y $4,8 \mathrm{~cm}$ ), necesitando a priori 2 clips a nivel de A2-P2. OI sin trombos, con medidas compatibles con un dispositivo 31 mm Amplatzer Amulet. Dada falla renal se decide no realizar scanner.

El procedimiento se realiza bajo anestesia general y asistido por ETE, con cceso venoso femoral derecho con punción eco-guiada. y heparina para ACT entre 300-350 segundos. Se realiza pre-cierre con 2 Perclose Proglide (Abbott Vascular, Santa Clara, CA, USA). Dado la necesidad de un procedimiento concomitante, se decide realizar una punción transeptal posterior y superior (mínimamente superior, 3,5- $4 \mathrm{~cm}$ ) (Figura 1A-B-C), facilitando técnicamente el implante del MC y posteriormente continuar con el LAAC. Luego de posicionar guía Amplatz (Boston Scientific) en vena pulmonar superior izquierda, se avanza cateter guía $24 \mathrm{~F}$. Se posicionan 2 clips en porción A2-P2 (primero el medial y luego el de la porción media-lateral) sin incidentes (Figura 1D-E). Al control ETE se observa la correcta posición con disminución de insuficiencia mitral residual (1-2+ / 4+) y gradiente de $4 \mathrm{mmHg}$. Posteriormente, manteniendo la guía Amplatz en vena pulmonar superior izquierda, se retira introductor $24 \mathrm{~F}$ del MC y se intercambia por un introductor $18 \mathrm{~F}$, ajustando el orificio venoso con los Perclose Proglide para disminuir el sangrado peri-introductor.

Luego, se avanza cateter guía 14F (Figura 1F). Se implanta Amplatzer Amulet $31 \mathrm{~mm}$ en OI con dificultad para lograr un buen alineamiento coaxial del dispositivo (Figura 2A-B-C). Luego multiples intentos, se logra una buena oclusión

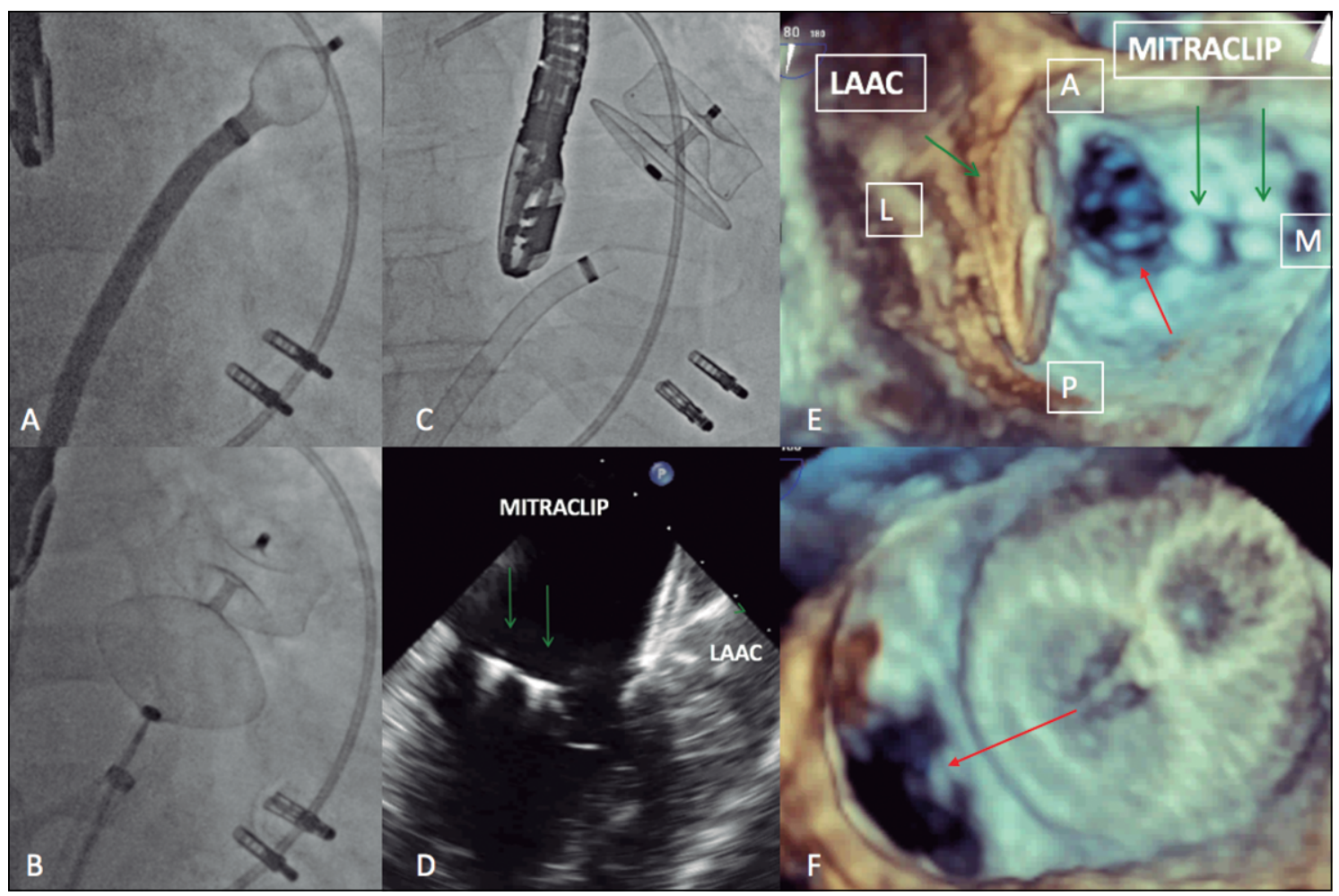

Figura 2. $L A A C$, implante, liberación e imágenes finales. A: Imagen angiográfica que muestra el momento de liberación inicial del dispositivo Amplatzer Amullet. Aunque con dificultad, se logra una buena alineación coaxial del dispositivo. B: Imagen angiográfica durante el Tug-Test. C: Liberación del dispositivo Amplatzer Amullet y su relación con los 2 dispositivos Mitraclip. D: Imagen de ETE donde muestra en flechas verdes los 2 Mitraclip liberados y a lateral el dispositivo de LAAC. E: Imagen de ETE 3D donde nos ayuda a entender el orientamiento espacial de los dos Mitraclip liberados en porcion medial del plano valvular (flechas verdes), el neo-orificio (flecha roja) y su relación con el dispositivo de LAAC. F: Imagen ETE 3D : porción lateral del plano valvular y su relación con el dispositivo Amplatzer, con el neo-orificio (flecha roja). 
de la orejuela confirmado por el ETE por lo que se libera sin incidentes (Figura 2D-E-F). Hemostasia femoral con cierre de los 2 Perclose Proglide.

Se decide alta con aspirina más clopidogrel. Seguimiento con ETE destaca una FEVI de 35\%, una insuficiencia mitral residual $2+/ 4+$ sin leak peri dispositivo Amplatzer Amulet y ausencia de shunt interatrial. En el seguimiento, paciente asintomática sin nuevas hospitalizaciones, con mejoría de la calidad de vida y con buena tolerancia al ejercicio moderado. Se obtuvo consentimiento de la paciente para este reporte clínico.

\section{Discusión}

La incidencia concomitante de IM moderada-severa y FA es variable según diferentes estudios con una frecuencia entre 32,7 y el $71,6 \%$;,5, lo que implica que al menos $1 / 3$ a $2 / 3$ de los pacientes probablemente tengan esta concomitancia.

La selección de los pacientes, que cumplan criterios clínicos y técnicos, es indispensable para obtener mejores resultados. El estudio incluye un ETE 3D y si es posible un AngioTAC gatillado para evaluar la factibilidad de ambos procedimientos y obtener una mejor orientación espacial, permitiendo un abordaje adecuado, la selección de los materiales y el lugar óptimo para la punción transeptal.

En el caso de LAAC, usualmente el orificio de entrada se ubica directamente frente a la fosa oval con una orientación anterior y superior. Se recomienda una punción transeptal infero-posterior que facilita el alineamiento coaxial. Una punción muy alta puede dificultar enormente la entrega del dispositivo por no lograr la coaxialidad necesaria para una buena oclusión, generando así tasas de éxito menores ${ }^{7}$.

En cuanto al implante de MC, se recomienda una punción supero-posterior y a una distancia mínima de la válvula mitral para poder maniobrar el dispositivo de entrega . En el caso de la IM primaria debe ser al menos $4-5 \mathrm{~cm}$ por sobre el anillo mitral y de al menos $3,5 \mathrm{~cm}$ sobre el plano anular, en los casos de IM secundaria donde la dilatación ventricular hace que el plano de coaptación de los velos sea más inferior ${ }^{8}$. Para ambos procedimientos, en forma concomitante, se recomienda una punción posterior y una altura lo más baja posible para realizar el implante del MC, dándole prioridad a este por sobre la LAAC.

La combinación de ambas intervenciones en un solo procedimiento es una alternativa atractiva tanto por comodidad del paciente, ya que desde un punto de vista técnico puede ser realizado en una misma hospitalización, requiriendo de un solo sitio de punción femoral y uno transeptal. Aunque las complicaciones por punción transeptal asistida por ecografía son bajas, un menor número de punciones disminuiría el daño residual en el septum ${ }^{9}$. Además el tiempo de anticoagulación oral posterior sería más breve, con menores tasas de sangrado y mortalidad tanto intrahospitalaria como en el seguimiento ${ }^{10}$.

El orden en que deben ser realizados ambos procedimientos no está del todo claro. Una serie publicada por Francisco et al. ${ }^{11}$, con implante de MC y del dispositivo de LAAC (Watchman, Boston Scientific), plantea realizar primero el cierre de orejuela ya que permite un incremento secuencial en el diámetro de las vainas, disminuyendo teóricamente el riesgo de sangrado del acceso vascular y el trauma sobre el SIA. Además, el dispositivo pudiese servir como referente anatómico para el implante del MC. No obstante, esta disposición podría correr el riesgo que el sistema de clipaje interfiriera con el LAAC recientemente implantado.

En el caso opuesto, comenzar con el implante del MC, implicaría cambiar por un introductor de menor tamaño lo cual pudiese dificultar la hemostasia. Se recomienda la vaina $18 \mathrm{~F}$ si se considera este orden de implante, controlando el sangrado con la postura inicial de 2 sistemas Perclose Proglide y permitiendo ajustar el orificio en la vena femoral al introductor que se esté utilizando, como se describió en nuestro caso clínico

Kuwata et al. ${ }^{9}$, reporta una serie de 25 pacientes tratados con esta técnica combinada y los comparó con igual número de sujetos sometidos solamente al implante de MC. A 30 días, el éxito de ambos procedimientos fue similar sin diferencias en la duración de la estadía hospitalaria. Tampoco hubo diferencias respecto a mortalidad intrahospitalaria, falla renal o eventos hemorrágicos. Sí hubo mayor duración del procedimiento y tiempo de radioscopía en el grupo combinado. Cabe destacar que en esta serie, el sitio de punción alto para MC no fue impedimento para lograr un éxito en el cierre de orejuela. 


\section{Conclusión}

En pacientes seleccionados, con IM severa y FA de alto riesgo, el tratamiento con $\mathrm{MC}$ asociado a cierre de OI, por vía transeptal es una técnica factible, segura y efectiva. La adecuada selección del paciente, así como una planificación del procedimiento es fundamental para un resultado exitoso.

\section{Referencias}

1. Cullen MW, Stulak JM, Li Z, Powell BD, White RD, Ammash NM, et al. Left Atrial Appendage Patency at Cardioversion After Surgical Left Atrial Appendage Intervention. Ann Thorac Surg 2016; 101 (2): 675-81.

2. Maisano F, Franzen O, Baldus S, Schäfer U, Hausleiter J, Butter C, et al. Percutaneous mitral valve interventions in the real world: Early and 1-year results from the ACCESS-EU, A prospective, multicenter, nonrandomized post-approval study of the MitraClip therapy in Europe. J Am Coll Cardiol 2013; 62 (12): 1052-61.

3. Stone GW, Lindenfeld JA, Abraham WT, Kar S, Lim DS, Mishell JM, et al. Transcatheter Mitral-Valve Repair in Patients with Heart Failure. N Engl J Med 2018; 379 (24): 2307-18.

4. January CT, Wann LS, Calkins H, Chen LY, Cigarroa JE CJ. 2019 AHA/ACC/HRS Focused Update of the 2014 AHA/ACC/HRS Guideline for the Management of Patients With Atrial Fibrillation: A Report of the American
College of Cardiology/American Heart Association Task Force on Clinical Practice Guidelines and the Heart. Circulation. 2019. Epub ahead of print doi:10.1161/ CIR.0000000000000665.

5. Feldman T, Foster E, Glower D, Kar S RM. Percutaneous Repair or Surgery for Mitral Regurgitation. N Engl J Med 2011; 364 (15): 1395-406.

6. Obadia J-F, Messika-Zeitoun D, Leurent G, Lung B. Percutaneous Repair or Medical Treatment for Secondary Mitral Regurgitation. N Engl J Med 2018; 379 (24): 2297-306.

7. Meier B, Blaauw Y, Khattab AA, Lewalter T. EHRA/ EAPCI expert consensus statement on catheter-based left atrial appendage occlusion. EuroIntervention 2015; 10 (9): 1109-25.

8. Gregory G, Shaw T, Gambra H BP. Cardiac Transseptal Catheterisation. PCR EAPCI Textbook Percutaneous Interv Cardiovasc Med 2015; 1 (1): Part 1. Online: https://www.pcronline.com/eurointervention/textbook/ pcr-textbook/chapter/current/69.html.

9. Kuwata S, Taramasso M, Zuber M. Feasibility of concomitant MitraClip and left atrial appendage occlusion. EuroIntervention 2017; 12 (16): 1940-5.

10. Taramasso M, Maisano F, Nietlispach F. TAVI and concomitant procedures: From PCI to LAA closure. EuroIntervention 2015; 11: W96-W100.

11. Francisco ARG, Infante de Oliveira E, Nobre Menezes M. Combined MitraClip implantation and left atrial appendage occlusion using the Watchman device: A case series from a referral center. Rev Port Cardiol 2017; 36 (7): 525-32. 